\title{
Non-invasive diagnosis for acute rejection using blood mRNA signature reflecting allograft status in kidney transplantation
}

\author{
Ahrim Han ${ }^{1}$, Jung-Woo Seo ${ }^{2}$, Yang Gyun Kim ${ }^{1}$ Ju-Young Moon ${ }^{1}$, Sang-Ho Lee ${ }^{1}$ \\ ${ }^{1}$ Department of Internal Medicine-Nephrology, Kyung Hee University Hospital at Gangdong, Seoul, Korea \\ ${ }^{2}$ Department of Core Research Laboratory, Medical Science Institute, Kyung Hee University Hospital at Gangdong, Seoul, Korea
}

Background: Despite improvements in immunosuppressive therapy over the years, acute rejection (AR) episodes that required treatment are still a significant risk factor for poor graft outcomes. Monitoring renal graft status through peripheral blood (PB) rather than invasive biopsy could reduce bleeding risk and costs.

Methods: Blood gene biomarker panels were discovered by microarrays and subsequently validated and cross-validated by qPCR. A total of 112 human PB samples, each paired with a graft biopsy, were analyzed ( 58 AR, 42 stable, and 12 other causes of graft injury). The differentially expressed genes by microarray, Q-PCR analysis of a four gene-set (GRB10, LGALS3BP, OLR1, and RNASE2) classified AR.

Results: We developed AR prediction model with the blood mRNAs by a binary logistic regression, and the AUC of the model was 0.76 in the training set. In addition, the decision curve analysis indicated a range of reasonable threshold probabilities for biopsy. Conclusions: Therefore, we suggest blood mRNA signature may serve as a non-invasive monitoring tool of AR for a clinical application and can assist with deciding whether to perform a biopsy in a recipient with a rise in creatinine and probably justifies a biopsy.

Corresponding author: Sang-Ho Lee

E-mail: Ishkidney@khu.ac.kr

(C) The Korean Society for Transplantation

This is an Open Access article distributed under the terms of the Creative Commons Attribution Non-Commercial License (http://creativecommons.org/licenses/by-nc/4.0/) which permits unrestricted non-commercial use, distribution, and reproduction in any medium, provided the original work is properly cited. 